\title{
MedienPädagogik
}

Zeitschrift für Theorie und Praxis der Medienbildung

www.medienpaed.com

ISSN 1424-3636

Jahrbuch Medienpädagogik 17:

Lernen mit und über Medien in einer digitalen Welt

Herausgegeben von Klaus Rummler, Ilka Koppel, Sandra Aßmann,

Patrick Bettinger und Karsten D. Wolf

\section{Lernen mit immersiver Virtual Reality: Didaktisches Design und Lessons Learned}

Josef Buchner und Diane Aretz

\section{Zusammenfassung}

In diesem Beitrag wird ein didaktisches Design zum Einsatz von mobiler immersiver Virtual Reality vorgestellt. Die Erprobung erfolgte im Mai 2019 an einer deutschen Grundschule. Die Schülerinnen und Schüler haben dazu Karton-VRBrillen selber zusammengebaut, getestet und im Anschluss für das Lernen eines Themas aus dem Sachunterricht verwendet. Die theoretische Grundlage für den Ablauf des Lernarrangements stellt die Generative-Learning-Theory dar. Diese sieht vor, dass die Lernenden während der Auseinandersetzung mit einem Medium Lernaktivitäten ausführen. Wir haben diese Vorgehensweisen noch um kommunikative und kollaborative Elemente erweitert, sodass die Kinder in Paaren gemeinsam an den Aufgabenstellungen arbeiteten. Auf eine Phase des «Sehens in der virtuellen Welt» folgte stets eine Phase des sozialen Austauschs mit dem Partner bzw. der Partnerin. Schriftliche und visuelle Zusammenfassungen wurden in einem «Team-Heftchen» festgehalten. Nach dem Lernereignis wurden die ermittelten Informationen in der Klasse gemeinsam besprochen sowie die von den Schülerinnen und Schülern kreierten eigenen Ideen für VR-Aufgabenstellungen präsentiert. Am nächsten Schultag erfolgte zudem eine Reflexion über das Medium VR. Lessons Learned sowie Empfehlungen zum Einsatz runden den Beitrag $a b$. 


\title{
Learning with Immersive Virtual Reality: Instructional Design and Lessons Learned
}

\begin{abstract}
In this paper a didactic framework for the use of mobile immersive virtual reality is presented. It was tested in May 2019 at a German primary school. The pupils assembled and tested cardboard VR glasses themselves and then used them to learn a topic from social studies and science. The theoretical basis for the learning arrangement is the Generative Learning Theory. This provides for the learners to carry out learning activities during their involvement with a medium. We have added communicative and collaborative elements to this approach so that the children worked together in pairs on the tasks. A phase of «seeing in the virtual world» was always followed by a phase of social exchange with the partner. Written and visual summaries were recorded in a "team booklet». After the learning event, the information obtained was discussed together in class and the students' own ideas for VR tasks were presented. On the next school day there was also a reflection on the medium VR. Lessons Learned as well as recommendations for future use summarize the contribution.
\end{abstract}

\section{Einleitung}

Die Diskussion, ob digitale Medien und Technologien in Bildungseinrichtungen eingesetzt werden sollten, wird auch in den 2020er Jahren nach wie vor geführt. Damit einher geht die Annahme, dass Medien oder Technologien bestimmte Wirkungen auslösen können und somit entscheidend für gelingendes Lernen seien. Dies ist erstaunlich, gibt es doch bereits viele Jahre, auch im deutschsprachigen Raum, Forschungsdisziplinen, die sich ausschliesslich mit den Gelingensbedingungen von Lehr- und Lernkontexten beschäftigen. Eine davon ist die Mediendidaktik, in der ganz konkret darüber nachgedacht wird, wie ein begründeter und geplanter Medieneinsatz zu anderem Lehren und Lernen führen kann (vgl. Kerres 2018). Die Frage ist also das Wie, nicht das Ob. Längst leben wir in einer mediatisierten Welt, mit der wir ständig im interaktiven Austausch stehen. Digitale Technik kann und sollte nicht mehr additiv zu klassischen 
Kulturtechniken hinzugedacht, sondern als integraler Bestand auf allen Ebenen des Bildungswesens ganz selbstverständlich mit-gedacht werden (für eine ausführliche Diskussion dazu siehe Kerres 2020 in diesem Buch).

Mit dem Aufkommen relativ «neuer» Technologien beginnt dann die Diskussion wieder von vorne. Aktuell gilt dies besonders für den Einsatz von Virtual Reality. Dem Lernen und Lehren mit und durch virtuelle Realitäten werden gar revolutionäre Potentiale zugeschrieben, die unsere Vorstellung von Bildung von Grund auf verändern werden (Rogers 2019).

Dass es nicht ganz so einfach ist, zeigen bereits erste Metaanalysen und Review-Studien. So wurden zwar oftmals positive Wirkungen auf den Lernerfolg festgestellt, vielfach jedoch erneut in klassischen Medien-Vergleichs-Studien (vgl. Krokos, Plaisant, und Varshney 2018).

Was fehlt sind didaktische Konzepte, Bezüge zu Lerntheorien oder Lernstrategien sowie eine detaillierte Beschreibung der Lernaktivitäten und verwendeten Materialien (Radianti et al. 2020; Jensen und Konradsen 2018; Wang et al. 2018).

Wir möchten in diesem Beitrag diesen Umstand aufgreifen und ein didaktisches Design zum Einsatz von immersiver VR zur Diskussion stellen. Das Design wurde im Mai 2019 an einer deutschen Grundschule erprobt. Unser Beitrag beginnt mit einer Schärfung des Begriffs Virtual Reality, stellt im Anschluss bereits vorhandene Erkenntnisse zum Einsatz von immersiver VR vor. Anknüpfend berichten wir unsere didaktische Analyse und geben einen Überblick über das von uns vorgeschlagene didaktische Design. Der Beitrag endet mit einer Diskussion des Vorgehens, den bereits angesprochenen Lessons Learned und wird schliesslich abgerundet durch ein Fazit.

\section{Was ist Virtual Reality?}

Virtual Reality (VR) wird definiert als gänzlich computer-generierte Welt, die von Menschen als Simulation der Realität wahrgenommen wird, möglichst viele Sinnesmodalitäten anspricht und die mithilfe von Eingabegeräten (mit)gestaltet und verändert werden kann (Burdea und Coiffet 2003, 3; Tao Ni et al. 2006). Damit unterscheidet sich VR von oftmals als Mixed Reality bezeichneten Technologien wie Augmented Reality (AR) und 
Augmented Virtuality (AV). Diese lassen sich auf dem von Milgram und Kishino (1994) aufgestellten Realitäts-Virtualitäts-Kontinuum (Abbildung 1) zwischen den beiden Endpunkten ansiedeln, VR hingegen markiert einen solchen.

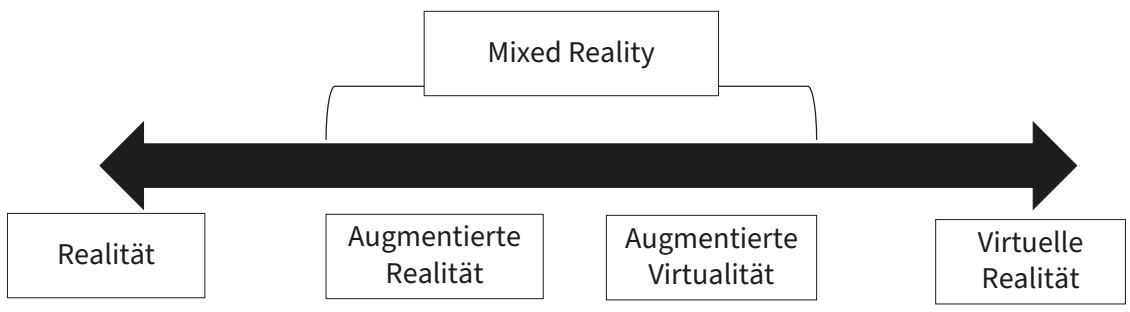

Abb. 1.: Das Realitäts-Virtualitäts-Kontinuum. Eigene Darstellung, angelehnt an Milgram und Kishino (1994).

Mittlerweile lassen sich für jede auf dem Kontinuum abgebildete Technologie unterschiedliche Formen bzw. Typen charakterisieren (Jung, Lee, und Biocca 2014, 214). Für VR-Anwendungen ist insbesondere die Abgrenzung zu eher traditionellen Computerschnittstellen von grosser Bedeutung. Unterschiede sind die egozentrische Perspektive, 3D-Interaktionen per Körperbewegungen und -gesten sowie der immersive Charakter der VR-Präsentation. Letztgenanntes wird in der Literatur als das zentrale technologische Merkmal von VR angesehen, heute bekannt unter dem Begriff der Immersion (Dörner et al. 2019, 14-15). Neben dieser technischen Anforderung ist als zweites zentrales Merkmal das psychologische Gefühl des Präsent-sein in der virtuellen Welt zu nennen, bezeichnet als Presence (Slater und Wilbur 1997; Slater und Sanchez-Vives 2016). Da die Begriffe in der Literatur immer wieder missverständlich und uneinheitlich verwendet werden (vgl. Dörner et al. 2019, 14, 19), erfolgt im nächsten Schritt eine Bestimmung dieser beiden, die VR-Forschung prägenden, Begrifflichkeiten.

\subsection{Immersion}

Immersion beschreibt die technischen Voraussetzungen, damit die Sinneseindrücke von Nutzenden möglichst umfassend angesprochen werden und somit eine Illusion der Realität entsteht. Aktuell dominieren Sehen 
und Hören, manche VR-Systeme lassen die User sogar bereits fühlen oder auch riechen. Hinzukommt das Ausführungen von Bewegungen in so natürlicher Form wie möglich, z.B. durch Drehen oder Auf- und Abbewegen des Kopfes wird auch die Szenerie in der VR-Welt entsprechend angepasst (Slater und Sanchez-Vives 2016, 4-5).

Vier technische Eigenschaften von Ausgabegeräten sind für Immersion erforderlich (Slater und Wilbur 1997, 604-5; Dörner et al. 2019, 14):

- Inclusive: Sinneseindrücke sollen ausschliesslich durch den Computer generiert werden, die Nutzenden sind isoliert von der realen Umwelt.

- Extensive: Es werden so viele Sinneseindrücke wie möglich angesprochen.

- Surrounding: Die computersimulierte Welt soll Nutzende vollständig umgeben und nicht nur auf ein enges Sichtfeld beschränkt sein.

- Vivid: Lebendige Darstellung der Realität in der VR, z.B. Auflösung, Farben, auditive Elemente etc.

Sherman und Craig $(2003,9)$ bezeichnen diese technischen Merkmal auch als physikalische Immersion und unterscheiden dazu das mentale Immersionserleben. In der VR-Forschungscommunity hat sich zur Beschreibung dieses mentalen Gefühls des Anwesend-sein in einer virtuellen Welt der Begriff Presence etabliert.

\subsection{Presence}

Im Gegensatz zur Immersion wird Presence als ein psychologisches Konstrukt verstanden, welches subjektiv wahrgenommen wird. Es wird beschrieben als das Gefühl, sich in der virtuellen Welt anwesend bzw. präsent zu fühlen und als Konsequenz Handlungen zu setzen, die auch unter realen Bedingungen gezeigt werden (Sanchez-Vives und Slater 2005; International Society for Presence Research 2000).

Zwei Aspekte kennzeichnen Presence (Slater, Spanlang, und Corominas 2010; Slater 2009):

- Place Illusion - Ortsillusion: Das Gefühl, am durch das VR-System simulierten Ort zu sein und z.B. von einem Feuer zu flüchten (Spanlang et al. 2007) 
- Plausibility Illusion - Plausibilitätsillusion: Das Gefühl, die computergenerierten Ereignisse finden tatsächlich statt. Empirische Nachweise dafür finden sich bei Slater et al. (2006) im Rahmen einer virtuellen Neuauflage des Milgram-Experiment sowie bei Slater et al. (2013) im Zuge einer Studie zur Interaktion zwischen Versuchspersonen und gänzlich computersimulierten Figuren.

Presence und Immersion können korrelieren (Slater und Sanchez-Vives 2016, 5), ausschliesslich auf die technologischen Eigenschaften zurückzuführen lässt sich das Präsenzerleben jedoch nicht. Als subjektiv wahrgenommene Empfindung unterliegt es zusätzlich Persönlichkeitseigenschaften sowie emotionalen und kognitiven Prozessen (Vgl. Hofer 2019).

Andere Autoren und Autorinnen diskutieren weitere Aspekte des Präsenzerlebens, etwa den Grad an Aufmerksamkeit (e.g. Burdea und Coiffet 2003), sowie die Rolle von Immersion und Presence beim Lesen, Spielen oder Ansehen von Filmen (e.g. Sherman und Craig 2003).

Forschungsarbeiten zu Immersion und Presence haben wesentlich dazu beigetragen, dass heute zwischen verschiedenen VR-Typen unterschieden wird.

\subsection{VR TYpen}

Desktopbasierte Anwendungen werden als nicht-immersive VR-Systeme bezeichnet. Die Interaktion findet hier ausschliesslich über traditionelle Eingabegeräte wie Maus und Tastatur statt. Simulationen, Spiele und virtuelle Welten wie Second Life können diesem Typ zugeordnet werden (Lee und Wong 2014).

Semi-immersive VR-Anwendungen nutzen die Einfachheit von desktopbasierten Systemen, erweitern die Interaktion jedoch um realistische Eingabegeräte. Ein Beispiel hierfür wäre ein mit Lenkrad und Pedalen ausgestatteter Fahrsimulator (Bamodu und Ye 2013).

Immersive VR kommt nun der gegenwärtig in der Allgemeinheit anzutreffenden Vorstellung von Virtual Reality am nächsten. Zur Darstellung von immersiven VR-Applikationen werden spezielle Brillen oder HeadMounted-Displays (HMDs) benötigt. Meist werden diese noch ergänzt um handliche Controller, die beim Eintauchen in die virtuelle Simulation zu 
Händen werden und Nutzerinnen und Nutzer damit in gewohnter Weise computergenerierte Objekte bewegen lassen. Bereits in den 1960er Jahren wurde von Ivan Sutherland ein erstes HMD entworfen, das als Sword of Damocles (Sutherland 1968) nicht nur in die Geschichte einging, sondern die Grundlage für das von Jaron Lanier entwickelte Eyphone, und somit für alle aktuellen VR-Brillen, schuf (Slater und Sanchez-Vives 2016, 3; Virtual Reality Society 2013).

Mittlerweile hat die technologische Entwicklung solche Fortschritte gemacht, sodass auf gänzlich neue Wege der Zugang zu immersiven VREreignissen ermöglicht wird. So können z.B. 360-Videos, abgespielt auf handelsüblichen Smartphones, mithilfe brillen-ähnlicher Halterungen ${ }^{1}$ stereoskopisch betrachtet werden (Buchner und Andujar 2019, 92) und 3DSimulationen über einfache Karton-Brillen ${ }^{2}$ betreten und manipuliert werden (Cochrane 2016).

Durch die Reduktion der technischen Anforderungen sinkt auch der finanzielle Aufwand auf Seiten der Bildungseinrichtungen, um Lernarrangements mit Immersiver VR umzusetzen.

Warum sich solche Umsetzungen lohnen könnten, wird im nächsten Abschnitt zu den Potentialen von immersiver VR für das Lehren und Lernen diskutiert.

\section{VR und Bildung}

Eine Diskussion zu nicht-immersiven VR-Applikationen hat bereits ausführlich stattgefunden. So können digitalen Spielen, Simulationen und virtuellen Welten mittlere Effektstärken für den Lernerfolg attestiert werden. Weiters können solche Anwendungen motivierend wirken, Emotionen bei Lernenden ansprechen und Kompetenzen, z.B. räumliches Vorstellungsvermögen und kritisches Denken, fördern (Maas und Hughes 2020; Merchant et al. 2014; Wang et al. 2018).

Wir fokussieren uns in diesem Beitrag auf den Einsatz immersiver VRAnwendungen und beschreiben die möglichen Potentiale auf der Grundlage von Ergebnissen aus ersten Review-Studien (Jensen und Konradsen

1 z.B. Homido Mini VR Glasses.

2 z.B. Cardboard. 
2018; Radianti et al. 2020) und den von Schwan und Buder (2006) sowie Dede (2009) formulierten lernbezogenen Handlungsmöglichkeiten.

\subsection{Trainingswelten}

Immersive VR-Anwendungen fördern psychomotorische Fähigkeiten sowie prozedurales Wissen. Es können Arbeitshandlungen und spezifische Fertigkeiten eingeübt, trainiert und vielfach wiederholt werden, die in der Realität zu gefährlich oder zu kostenintensiv wären. Auch das Manipulieren von physikalischen Gesetzmässigkeiten im Sinne des Experimentierens ist denkbar. Wichtig ist bei solchen Trainingswelten, dass die Lernenden mit den Objekten in der virtuellen Welt interagieren können und ihre Handlungen zu spür- bzw. sichtbaren Ereignissen führen. Als Beispiel sei hier das Lackieren von Autobauteilen in einer virtuellen Werkstatt genannt (Sander 2019; Zender et al. 2019).

\subsection{Konstruktionswelten}

VR-Systeme erlauben Lernenden das Erstellen und Gestalten eigener virtueller Welten. In der internationalen Literatur finden sich aktuell noch wenige Studien, die VR-Konstruktionswelten untersuchten und damit VR an sich zum Lerngegenstand machten (Radianti et al. 2020, 23). Ein Beispiel für den Fremdsprachenunterricht beschreibt Wössner (2019). Ihre Schülerinnen und Schüler haben mit der Software Holobuilder $360^{\circ}$-Rundgänge mit Audio- und Fotodateien erstellt sowie mit der Applikation CoSpaces $E d u^{4}$ 3D-Räume programmiert und mit persönlichen Lernartefakten ausgestaltet. Auch das beliebte Computerspiel Minecraft verfügt mittlerweile um eine VR-Erweiterung. Lernende können so über Brillen ihre virtuellen Welten direkt in der VR anfertigen (Mojang 2019).

3 https://www.holobuilder.com.

4 https://cospaces.io/edu/. 


\subsection{Explorationswelten}

VR-Anwendungen mit explorativen Charakter erlauben den Lernenden sich an aktuell unzugänglichen (Lern)Orten zu bewegen und diese möglichst selbstgesteuert zu untersuchen. Dazu zählen virtuelle Ausflüge in der Geographie (Minocha, Tilling, und Tudor 2018), simulierte Reisen in die Vergangenheit (Bunnenberg 2018) oder auch Erkundungen des menschlichen Körpers (Parong und Mayer 2018). Gefördert wird meist deklaratives Wissen.

Dede (2009) weist im Zuge solcher VR-Systeme noch zusätzlich auf die Möglichkeiten des situierten und multiperspektivischen Lernens hin. Solche Lerngelegenheiten können dann auch zur Entwicklung affektiver Fähigkeiten, z.B. dem Empathie-Erleben, führen (e.g. Shin 2018). Voraussetzungen hierfür sind hoch-sensitive Simulationen der eigenen (körperlichen) Person oder sozialer Situationen (Jensen und Konradsen 2018, 1525).

Erweitert werden können diese Handlungsmöglichkeiten von Lernenden in VR-Systemen noch um instruktionspsychologische Grundlagen (Mayer 2019; Moreno und Mayer 2001) und klare Lernstrategien, wie etwa in Parong und Mayer (2018). Die Autorin und Autor haben in ihrer Studie eine VR-Anwendung zum Erlernen der menschlichen Anatomie aus der Perspektive der Generative-Learning-Theory (Fiorella und Mayer 2016) untersucht. Die Ergebnisse zeigen, dass Lernaktivitäten, wie das Anfertigen von Zusammenfassungen, während dem immersiven Lernen zu besseren Lernleistungen führen und sich nicht negativ auf das Motivationserleben auswirken (Parong und Mayer 2018, 794).

\section{Didaktisches Design zum Einsatz von Immersiver VR}

Unsere Konzeption orientiert sich an dem Ansatz der gestaltungsorientierten Mediendidaktik nach Kerres (2005) sowie dem Rahmenmodell der Mediendidaktik (Kerres 2018, 229).

Der Einsatz eines Mediums soll demnach ein Bildungsanliegen adressieren und zur Lösung dieses Anliegens beitragen. Dazu ist eine didaktische Analyse notwendig (Kerres 2005a, 4-5), die schliesslich zu unserem didaktischen Design führt. 


\subsection{Bedingungen und Zielgruppe}

Der Einsatz soll an einer deutschen Grundschule realisiert werden. Die Schule hat eine Medienbeauftragte, die das Kollegium mit Informationen rund um das Thema Lernen mit Medien versorgt. Eine Moodle-Plattform mit mehreren Kursen ist vorhanden, ebenso Schüler-Tablets, die von den Lehrpersonen ausgeliehen und im Unterricht eingesetzt werden können.

Die Schülerinnen und Schüler haben bereits mit digitalen Geräten im Unterricht gearbeitet und bei Bedarf können sie auch ein Smartphone, das eigene oder jenes der Eltern in Absprache mit diesen, in die Schule mitbringen. Im Regelfall ist ein Mitbringen des Smartphones in die Schule nicht gestattet.

Die Eltern stehen einem Einsatz von digitalen Medien für Lehr- und Lernzwecke positiv gegenüber, ebenso die Schulleitung.

\subsection{Lehrinhalte und -ziele}

Die Unterrichtsreihe aus dem Sachunterricht verfolgte das Ziel, die Lebensgewohnheiten früherer Kulturen und Epochen mit heutigen Lebensgewohnheiten zu vergleichen (QUA-LiS NRW 2017). Zudem sollte eine kritische Auseinandersetzung mit VR als Medium erfolgen, sodass nicht nur mit, sondern auch über Medien gelernt wird (Peschel 2016).

\subsection{Methode(n)}

Lernen in der Grundschule soll Möglichkeiten bieten, ganzheitlich zu lernen, gemeinsam an Aufgaben zu arbeiten und zur Wissensgenerierung selbstbestimmt Lernwege zu gehen (vgl. Ministerium für Schule und Weiterbildung NRW 2016). Wir versuchen in unserer Konzeption diesem Anspruch gerecht zu werden, indem wir zunächst eine Phase im Plenum planen, in der jeder und jede eigenständig eine Karton-VR-Brille zusammenbaut. Im Anschluss erfolgt das Testen der Brillen in Teams. Als $\mathrm{Me}-$ thode für die Phase der Wissensakquirierung orientieren wir uns in Anlehnung an Parong und Mayer (2018) an der Generative-Learning-Theory. Das Grundprinzip dieser Theorie sieht vor, dass über Aktivitäten wie das Zusammenfassen, Zeichnen oder Erklären, neue Wissensinhalte mit dem 
Vorwissen verknüpft werden und später auf neue Aufgaben angewandt werden können (Fiorella und Mayer 2016). Wir erweitern diese Theorie um die Elemente Kommunikation und Kollaboration, d.h. die Aktivitäten werden stets im Austausch mit einem Partner/einer Partnerin durchgeführt und Lernartefakte wurden gemeinsam gestaltet.

\subsection{Medien}

Das Ziel der Klassenlehrerin ist, über immersive VR den Schülerinnen und Schülern einen möglichst authentischen Eindruck eines klassischen deutschen Haushalts zu vermitteln. Dazu werden $360^{\circ}$-Aufnahmen einer Wohnung angefertigt, die mithilfe der proprietären Software 3 DVista $^{5}$ zu interaktiven und stereoskopisch zu betrachtenden Szenen konfiguriert werden. Interaktiv bedeutet hier, dass durch das In-den-Blick-nehmen eingefügter Symbole (Abbildung 2) Bewegungen erzeugt und in die Szene eingespielte digitale Artefakte abgespielt bzw. geöffnet werden können. Verharrt der Blick etwa für wenige Sekunden auf der Tür, verlässt man den Raum und betritt den nächsten. Ebenfalls zu sehen ist in Abbildung 2 die stereoskopische Ansicht, so wie sie auch die Schülerinnen und Schüler gesehen haben. Drehen die Lernenden ihren Kopf nach links und rechts bewegt sich das $360^{\circ}$-Bild mit. So war eine Untersuchung des ganzen Raumes möglich. Diese Egoperspektivität ist ein wichtiger Baustein für das Immersionserleben (siehe 2.1). Möglich macht dies das Abspielen der Szenerie im Browser eines Smartphones bei waagerechter Positionierung des Geräts.

5 http://www.3dvista.com. 


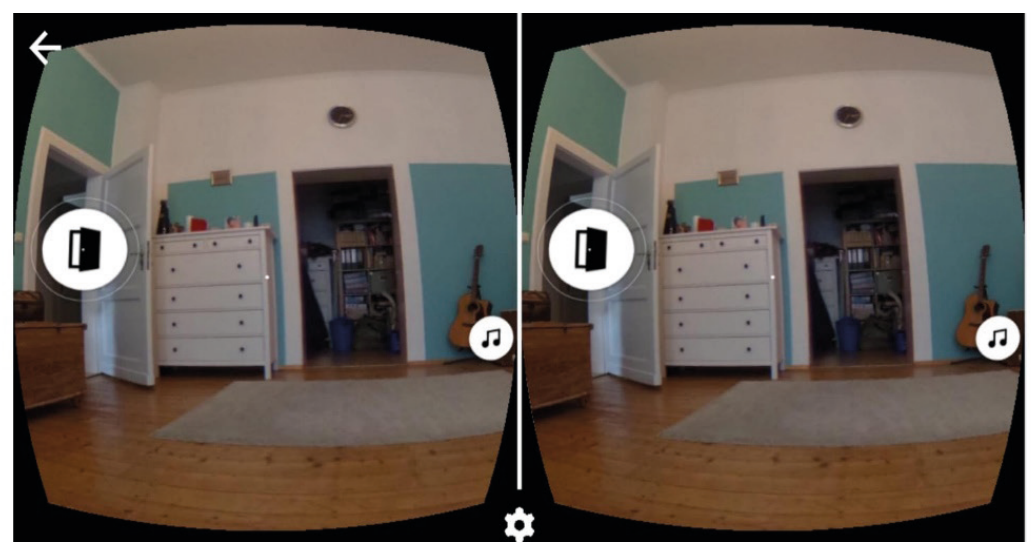

Abb. 2.: Stereoskopische Ansicht wie in der Karton-VR-Brille mit Tür- und Notenschlüsselsymbol.

Die Lernaktivitäten werden in einem «Team-Heftchen» festgehalten (Abbildung 3).
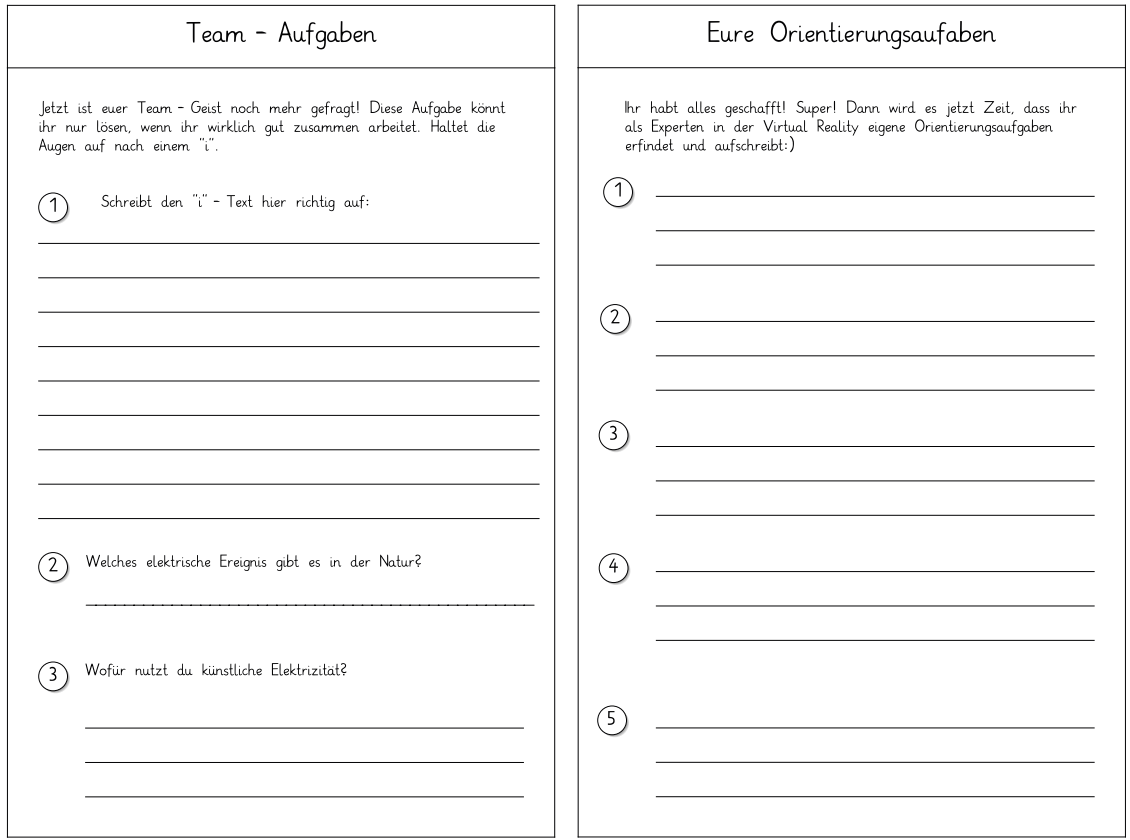

Abb. 3.: Auszug zweier Seiten aus dem begleitenden Team-Heftchen. 


\subsection{Sehen in der virtuellen Welt, sprechen, zuhören und ge- stalten in der realen Welt}

Abbildung 4 zeigt eine Übersicht unser didaktisches Design zum Einsatz von immersiver VR im Grundschulunterricht. Erprobt wurde es im Mai 2019.

Beim Zusammenbauen der Karton-VR-Brillen war die Klassenlehrerin und eine zweite Lehrerin anwesend. Das Zusammensetzen der Brillen wurde Schritt für Schritt angeleitet. Der Prozess wurde mithilfe der Tablet-Kamera und dem Beamer auf einer Leinwand live übertragen.

Im Anschluss an den Herstellungsprozess testeten die Kinder ihre Brillen mit verschiedenen Anwendungen, z.B. $360^{\circ}$-Videos von YouTube.

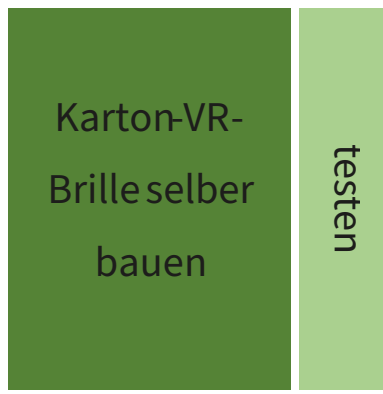

\section{Sehen in der}

$$
\text { virtuellen Welt - }
$$

Sprechen, zuhören

und gestalten in

der realen Welt

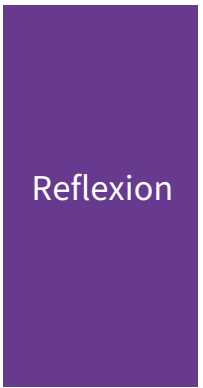

Abb. 4.: Übersicht des didaktischen Designs.

Diese Phase dauert ca. eine Unterrichtseinheit. Im Anschluss folgte der Kern unseres Lernarrangements, das Sehen in der virtuellen Welt mithilfe der Karton-VR-Brillen, stets gefolgt von einer Phase des Austausch mit dem Partner oder der Partnerin. Das Gesehene wurde im Heftchen schriftlich zusammengefasst und/oder visuell festgehalten z.B. als Mind-Map. Abbildung 5 zeigt zwei Lernende im Augenblick des Sehens in der virtuellen Welt. Am Tisch erkennbar sind die «Team-Heftchen» sowie Tablets für weitere Recherchemöglichkeiten. 


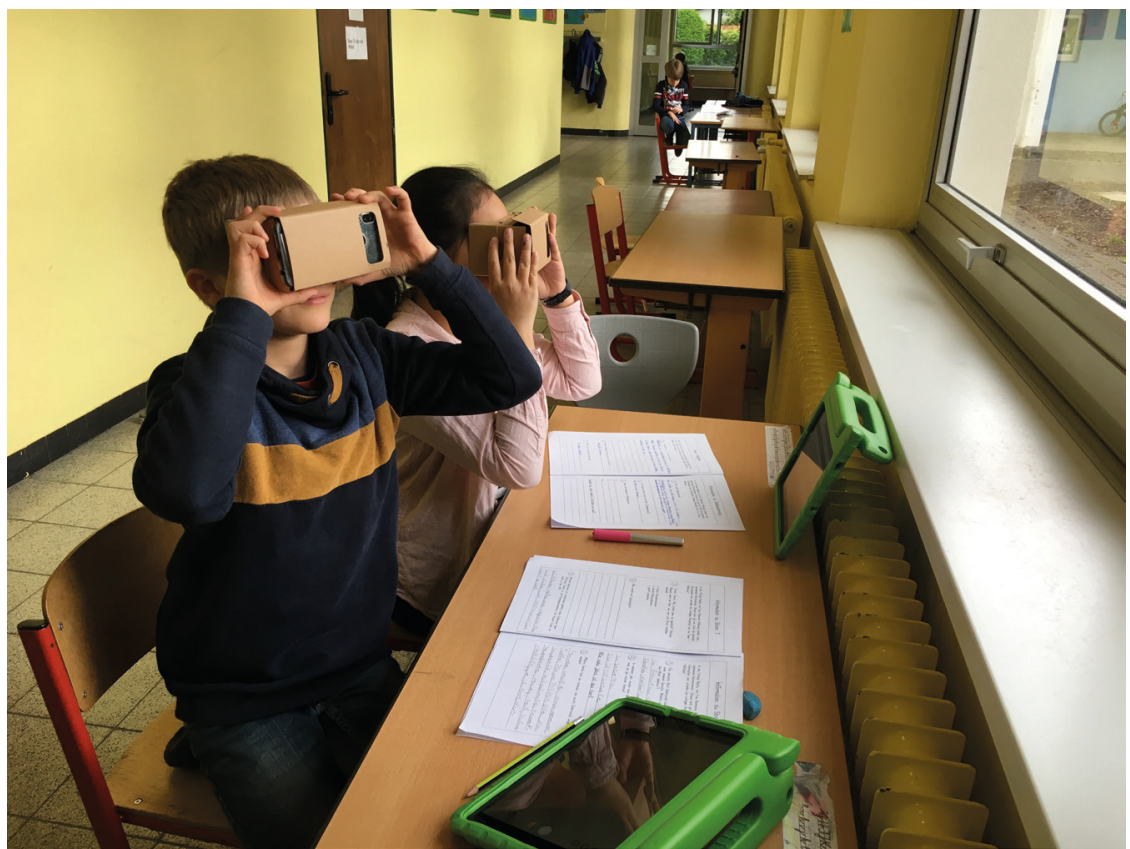

Abb. 5.: Zwei Lernende mit den Karton-VR-Brillen.

Diese intensive Lernphase dauerte ca. zwei Unterrichtsstunden. In der Kontrollphase wurden die Ergebnisse aus den Heftchen besprochen und die Schülerinnen und Schüler bekamen die Gelegenheit, ihre erdachten VR-Lernaufgaben in der Klasse zu präsentieren.

Die Reflexion des Erlebten konnte erst am nächsten Schultag durchgeführt werden, da der Schulunterricht nach den Präsentationen zu Ende war.

\section{Diskussion}

Für die meisten Kinder war das Eintauchen mit der zuvor selbst gebastelten Karton-VR-Brille die erste Erfahrung mit immersiver VR, Probleme mit Motion Sickness, also Schwindel oder Übelkeit, wurden uns nicht rückgemeldet.

Unser Ansatz Sehen in der virtuellen Welt, sprechen, zuhören und gestalten in der realen Welt darf nicht als allgemeingültiges Prinzip verstanden werden. Wir sind uns sehr bewusst, dass mit entsprechenden 
VR-Anwendungen wahrscheinlich alle von uns gestellten Lernaufgaben und -aktivitäten auch in einer konstruierten virtuellen Welt zu bearbeiten wären. Aus technischer Sicht wäre der Aufwand jedoch um ein Vielfaches höher als mit der von uns beschriebenen mobilen Variante für immersive VR. Um dennoch die Potentiale von VR für eher konstruktivistisches Lernen einzulösen, also den Lernenden Handlungsmöglichkeiten anzubieten, die über das reine explorieren hinausgehen (vgl. Hellriegel und Čubela 2018), haben wir kommunikative und kooperative Elemente ausserhalb der VR als fixe Lernereignisse in den jeweiligen Teams eingeplant. In der VRUmgebung selbst hatten die Kinder zumindest die Möglichkeit sich frei zu bewegen, Räume und Objekte selbstgesteuert aufzusuchen bzw. auszuwählen und im eigenen Tempo vorzugehen. Erwähnenswert ist auch, dass die Kinder natürlich begeistert von diesem «anderen» Unterricht waren (vgl. ebd., 65-66).

Aus unseren subjektiven Beobachtungen können wir schliessen, dass der Einsatz der Lernstrategien aus der Generative-Learning-Theory auch bei unserer Umsetzung nicht zu einer Verringerung der Motivation führte. Auch der Lernerfolg wurde in der Kontrollphase erkennbar. Die Schülerinnen und Schüler hatten fast alle Aufgaben im Heftchen gelöst und in ihren Präsentationen eine Vielzahl an Ideen für weitere VR-Lernaktivitäten dargeboten. Im nächsten Schritt wäre nun geplant, diese Ideen auch umzusetzen, etwa mit dem in Hellriegel und Čubela $(2018,70)$ beschriebenen «Google Expeditions-Tour Creator».

Anzumerken ist noch, dass besonders bei $360^{\circ}$-Medien die fehlenden Interaktions- und Steuerungsmöglichkeiten kompensiert werden müssen, möchte man dem Anspruch an immersive VR-Welten zumindest annähernd gerecht werden. Auch das haben wir in unserem Design versucht. Technisch mithilfe der interaktiven Symbolen und der egozentrisch-stereoskopischen Perspektive, didaktisch mit den Aufgabenstellungen und Aktivitäten, dem sozialen Austausch und dem Gestalten von eigenen Lernartefakten mit Papier und Stift.

$\mathrm{Ob} 360^{\circ}$-Bilder oder -Videos als VR bezeichnet werden können, hängt von diesen und den anderen in 2.1 und 2.2 aufgezählten Merkmalen immersiver VR-Anwendungen ab. Sind diese erfüllt, halten Slater und SanchezVives $(2016,35)$ eine Debatte darüber für überflüssig. 


\section{Empfehlungen und Lessons Learned}

Am Ende möchten wir noch ein paar Erfahrungen weitergeben, die anderen Lehrenden bei der Planung von immersiven Lernarrangements helfen können.

Ganz zentral ist für uns, dass die Schulleitung, Eltern und auch die Schülerinnen und Schülern über das Vorhaben informiert werden. Die Schulleitung kann bei der Beschaffung der notwendigen Mittel, z.B. den Karton-VR-Brillen unterstützen. Da für mobile immersive VR Smartphones benötigt werden, ist eine Absprache mit den Eltern zu empfehlen bzw. war in unserem Fall notwendig. Viele der Kinder haben noch kein eigenes Gerät und brachten für unser Vorhaben daher das Smartphone der Eltern mit in die Schule. Mit den Schülerinnen und Schülern sollten Regeln zum Smartphone-Einsatz vereinbart werden sowie das Vorgehen beim Einsatz von immersiver VR besprochen werden. Wir empfehlen nach dem Zusammenbauen eine Phase des Testens. Ein Kind erprobte die Brille mit einer VR-Anwendung, der Partner oder die Partnerin achtete darauf, dass keine gefährlichen Situationen entstanden oder könnten bei auftretendem Schwindelgefühl sofort eingreifen.

Weitere Lessons Learned sind:

Karton-VR-Brillen:

- Können günstig online bestellt werden. Oftmals gibt es solche Brillen auch als Werbegeschenke.

- Wenn ganze Sets erworben werden unbedingt darauf achten, dass die Linsen dabei sind.

- Genug Brillen kaufen! Beim Auspacken bzw. Zusammenbauen kann es schon mal passieren, dass Kartonteile kaputt gehen oder verschwinden.

- Vorlagen zum Basteln von VR-Brillen gibt es online (e.g. futurezone/PR/ JJ 2019). Ein Pizzakarton reicht als Material aus. Wichtig ist, dass die speziellen Linsen dann zusätzlich bestellt werden müssen.

- Trotz Einfachheit für das Zusammenbauen genug Zeit einplanen. Wir waren überrascht, wie lange es bei manchen gedauert hat bzw. auch wie anspruchsvoll manche Teilschritte waren. 


\section{Lernprozess:}

- Am Beginn Orientierungsaufgaben anbieten, sodass sie Lernenden die Handhabung der Technologie erlernen können.

- Lernaufgaben und -aktivitäten anbieten, die mithilfe des Gesehenen in der VR gelöst werden können/müssen.

- Team-Aufgaben so gestalten, dass sie auch wirklich nur im Austausch mit dem Partner/der Partnerin gelöst werden können.

- Zeit und Raum für das Lernen bereitstellen.

\section{Reflexion:}

- Hier gibt es online bereits gute Materialien zu VR, z.B.: MZM o. J.; Quast 2018; Jugend und Medien o. J.

- Zitate oder kontroverse Aussagen zum Einstieg bieten sich an. Anregungen finden sich in dem empfehlenswerten Artikel von Thomas Damberger (2017).

\section{Fazit}

Der Einsatz von immersiver VR hat sich auf alle Fälle gelohnt. Die Schülerinnen und Schüler haben ein «neues» Medium als Lerntechnologie kennengelernt und darüber nachgedacht, welche Auswirkungen und Konsequenzen virtuelle Welten für sie selber und die Gesellschaft haben könnten.

Andere Lehrpersonen der Schule können bei Bedarf nun die KartonVR-Brillen nutzen. Dies ist auch bereits geschehen. So haben ca. drei Wochen nach unserer Umsetzung über 60 Kinder einen Tauchgang im Ozean mithilfe der Karton-Brillen und der Vimeo-App (Oceans36o 2019) durchgeführt. Eingebettet war dies in ein klassenübergreifendes Projekt zu «Plastik im Meer». Auch hier waren die Kinder von der Möglichkeit des immersiven Taucherlebnisses ganz begeistert. Gleichzeitig konnten sie sehr real erleben, wie Teile des Ozeans mehr und mehr durch Plastikmüll verschmutzen. Als Konsequenz meldeten manche Eltern den Lehrpersonen zurück, dass nun beim gemeinsamen Einkaufen keine Plastikverpackungen mehr gekauft werden dürften. Ein erster Ansatzpunkt dafür, dass Karton-VRBrillen sogar affektive Lernereignisse anstossen können. 
Wir möchten mit der Beschreibung unserer Umsetzung von immersiver VR in der Praxis anderen Lehrpersonen Mut machen.

Es ist möglich, Kindern das Eintauchen in immersive Welten zu ermöglichen, dabei Spass zu haben und gleichzeitig Lernprozesse anzuregen.

Nur ein Mehr an didaktisch gut durchdachten und geplanten Umsetzungen kann dafür sorgen, dass auch das Medium «VR» entmystifiziert wird und schlussendlich als Bildungstechnologie wahrgenommen wird und in Schulen, Hochschulen und Weiterbildungseinrichtungen ankommt.

\section{Literatur}

Bamodu, Oluleke, und Xuming Ye. 2013. "Virtual Reality and Virtual Reality System Components». In Proceedings of the 2nd International Conference On Systems Engineering and Modeling, 4921-24.

Buchner, Josef, und Alberto Andujar. 2019. "The expansion of the classroom through mobile immersive learning». In Proceedings of the 15th International Conference Mobile Learning 2019, herausgegeben von Inmaculada Arnedillo Sanchez, Pedro Isaias, Pascal Ravesteijn, und Guido Ongena, 89-95. Utrecht, The Netherlands: iadis.

Bunnenberg, Christian. 2018. «Virtual Time Travels? Public History and Virtual Reality». Public History Weekly. https://public-history-weekly.degruyter. com/6-2018-3/public-history-and-virtual-reality/.

Burdea, Grigore C., und Philippe Coiffet. 2003. Virtual Reality Technology. 2nd Edition. Wiley-IEEE Press. https://books.google.de/books?id=oxWgPZbcz4AC.

Cochrane, Thomas. 2016. «Mobile VR in Education: From the Fringe to the Mainstream». International Journal of Mobile and Blended Learning 8 (4): 44-6o. https://doi.org/10.4018/IJMBL.2016100104.

Damberger, Thomas. 2017. «Bildungsreise in digitale Welten». Pädagogische Rundschau, 20.

Dede, Chris. 2009. «Immersive Interfaces for Engagement and Learning». Science 323 (5910): 66-69.

Dörner, Ralf, Wolfgang Broll, Bernhard Jung, Paul Grimm, und Martin Göbel. 2019. «Einführung in Virtual und Augmented Reality». In Virtual und Augmented Reality (VR/AR). Grundlagen und Methoden der Virtuellen und Augmentierten Realität, herausgegeben von Ralf Dörner, Wolfgang Broll, Paul Grimm, und Bernhard Jung, 2. Auflage, 1-42. Wiesbaden u.a.: Springer Vieweg. 10.1007/978-3662-58861-1.

Fiorella, Logan, und Richard E. Mayer. 2016. «Eight Ways to Promote Generative Learning». Educational Psychology Review 28 (4): 717-41. https://doi. org/10.1007/s10648-015-9348-9. 
futurezone/PR/JJ. 2019. «Virtual Reality für lau: So machst du dein Smartphone zur VR-Brille». 2019. https://www.futurezone.de/produkte/article215704365/ DIY-In-5-Schritten-zu-deiner-eigenen-VR-Brille.html.

Hellriegel, Jan, und Dino Čubela. 2018. «Das Potenzial von Virtual Reality für den schulischen Unterricht - Eine konstruktivistische Sicht». MedienPädagogik: Zeitschrift für Theorie und Praxis der Medienbildung, Dezember, 58-80. https:// doi.org/10.21240/mpaed/00/2018.12.11.x.

Hofer, Matthias. 2019. "The feeling of being there": Presence-Erleben als Folge kognitiver und emotionaler Verarbeitungsprozesse». In Emotions Meet Cognitions, herausgegeben von Holger Schramm, Jörg Matthes, und Christian Schemer, 53-62. Wiesbaden: Springer Fachmedien Wiesbaden. https://doi. org/10.1007/978-3-658-25963-1_4.

International Society for Presence Research. 2000. «The Concept of Presence: Explication Statement». International Society for Presence Research. 2000. https://ispr.info/about-presence-2/about-presence/.

Jensen, Lasse, und Flemming Konradsen. 2018. «A Review of the Use of Virtual Reality Head-Mounted Displays in Education and Training». Education and Information Technologies 23 (4): 1515-29. https://doi.org/10.1007/s10639-0179676-0.

Jugend und Medien. o. J. "Virtual Reality». Zugegriffen 30. November 2019. https://www.jugendundmedien.ch/de/medienkompetenz-foerdern/eltern-familie/virtual-reality.html.

Jung, Soyoung, Daeun Lee, und Frank Biocca. 2014. «Psychological Effects on 3 Dimensions Projection Mapping Versus 2 Dimensions: Exploratory Study». In Challenging Presence. Proceedings of the International Society for Presence Research, herausgegeben von Anna Felnhofer und Oswald D. Kothgassner, 21322. Wien, Austria: Facultas Verlag.

Kerres, Michael. in Press. «Bildung in der digitalen Welt: Über Wirkungsannahmen und sie soziale Konstruktion des Digitalen». MedienPädagogik: Zeitschrift für Theorie und Praxis der Medienbildung. https://www.researchgate.net/publication/340266940_Kerres-KonstruktDigitaleBildung_O_1.

Kerres, Michael. 2005a. «Didaktisches Design und E-Learning. Zur didaktischen Transformation von Wissen in mediengestütze Lernangebote.» In E-Learning. Eine multiperspektivische Standortbestimmung, herausgegeben von Damian Miller, 156-82. Bern u.a.: Haupt.

Kerres, Michael. 2005b. «Gestaltungsorientierte Mediendidaktik und ihr Verhältnis zur Allgemeinen Didaktik». In Allgemeine Didaktik im Wandel, herausgegeben von B. Dieckmann und P. Stadtfeld, 214-34. Bad Heilbrunn: Klinhardt Verlag. https://learninglab.uni-due.de/sites/default/files/mdidaktikkerres_o.pdf.

Kerres, Michael. 2018. Mediendidaktik: Konzeption und Entwicklung mediengestützter Lernangebote. 5. Auflage. Berlin: De Gruyter Oldenbourg Verlag. 
Kerres, Michael. 2020. "Bildung in der digitalen Welt: Über Wirkungsannahmen und die soziale Konstruktion des Digitalen». Herausgegeben von Klaus Rummler, Ilka Koppel, Sandra Aßmann, Patrick Bettinger, und Karsten D. Wolf. MedienPädagogik: Zeitschrift für Theorie und Praxis der Medienbildung, Jahrbuch Medienpädagogik (17): 1-32. https://doi.org/10.21240/mpaed/ jb17/2020.04.24.X.

Krokos, Eric, Catherine Plaisant, und Amitabh Varshney. 2018. «Virtual Memory Palaces: Immersion Aids Recall». Virtual Reality. https://doi.org/10.1007/ s10055-018-0346-3.

Lee, Elinda Ai-Lim, und Kok Wai Wong. 2014. «Learning with Desktop Virtual Reality: Low Spatial Ability Learners Are More Positively Affected». Computers \& Education 79 (Oktober): 49-58. https://doi.org/10.1016/j.compedu.2014.07.010.

Maas, Melanie J., und Janette M. Hughes. 2020. «Virtual, Augmented and Mixed Reality in K-12 Education: A Review of the Literature». TechnologY, PedagogY and Education, März, 1-19. https://doi.org/10.1080/1475939X.2020.1737210.

Mayer, Richard E. 2019. «Thirty Years of Research on Online Learning》. Applied Cognitive Psychology 33 (2): 152-59. https://doi.org/10.1002/acp.3482.

Merchant, Zahira, Ernest T. Goetz, Lauren Cifuentes, Wendy Keeney-Kennicutt, und Trina J. Davis. 2014. "Effectiveness of Virtual Reality-Based Instruction on Students' Learning Outcomes in K-12 and Higher Education: A MetaAnalysis». Computers \& Education 70: 29-40. https://doi.org/10.1016/j.compedu.2013.07.033.

Milgram, Paul, und Fumio Kishino. 1994. «A Taxonomy of Mixed Reality Visual Displays». IEICE Transactions on Information System 77 (12): 1321-29.

Ministerium für Schule und Weiterbildung NRW, Hrsg. 2016. Bildungsgrundsätze: mehr Chancen durch Bildung von Anfang an; Grundsätze zur Bildungsförderung für Kinder von o bis 10 Jahren in Kindertagesbetreuung und Schulen im Primarbereich in Nordrhein-Westfalen. Freiburg Basel Wien: Herder.

Minocha, Shailey, Steve Tilling, und Ana-Despina Tudor. 2018. «Role of Virtual Reality in Geography and Science Fieldwork Education». In Knowledge Exchange Seminar Series, Learning from New Technology. Belfast.

Mojang. 2019. «Minecraft in VR erkunden». Minecraft.net. 2019. https://www.minecraft.net/de-de/vr/.

Moreno, Roxana, und Richard E Mayer. 2001. «Virtual Reality and Learning: Cognitive and Motivational Effects of Students' Sense of Presence». In Proceedings of Human-Computer Interaction, 65-67. Mahwah, NJ.

MZM. o.J. «ECHT - Virtual Reality». https://www.medienzentrum-muc.de/angebotevent/echt-virtual-reality/.

Oceans360. 2019. «Oceans 360». Oceans 360. 2019. https://oceans360.org/.

Parong, Jocelyn, und Richard E. Mayer. 2018. «Learning Science in Immersive Virtual Reality.» Journal of Educational Psychology 110 (6): 785-97. https://doi. org/10.1037/eduo000241. 
Peschel, Markus. 2016. «Medienlernen im Sachunterricht - Lernen mit Medien und Lernen über Medien». In Neue Medien in der Grundschule 2.0, herausgegeben von Markus Peschel und Thomas Irion. Frankfurt am Main: Grundschulverband.

QUA-LiS NRW. 2017. «Schulentwicklung NRW - Lehrplannavigator Grundschule - Sachunterricht - Lehrplan Sachunterricht - 2 Bereiche und Schwerpunkte». 2017. https://www.schulentwicklung.nrw.de/lehrplaene/lehrplannavigator-grundschule/sachunterricht/lehrplan-sachunterricht/bereiche/bereiche. html.

Quast, Christina. 2018. "Neue Realitäten - Augmented Reality und Virtual Reality - Im Blickpunkt». 2018. https://imblickpunkt.grimme-institut.de/neuerealitaeten-augmented-reality-und-virtual-reality/.

Radianti, Jaziar, Tim A. Majchrzak, Jennifer Fromm, und Isabell Wohlgenannt. 2020. «A Systematic Review of Immersive Virtual Reality Applications for Higher Education: Design Elements, Lessons Learned, and Research Agenda». Computers \& Education 147 (April): 103778. https://doi.org/10.1016/j.compedu.2019.103778.

Rogers, Sol. 2019. «Virtual Reality: THE Learning Aid Of The 21st Century». 2019. https://www.forbes.com/sites/solrogers/2019/03/15/virtual-reality-the-learning-aid-of-the-21st-century/\#7bf34e5fi39b.

Sanchez-Vives, Maria V., und Mel Slater. 2005. «From Presence to Consciousness through Virtual Reality». Nature Reviews Neuroscience 6 (4): 332-39. https:// doi.org/10.1038/nrn1651.

Sander, Pia. 2019. "Das 4C/ID-Modell zum Training komplexer kognitiver Fertigkeiten». Webinar gehalten auf der VR/AR-Learning. Gemeinsamer Arbeitskreis der GI-Fachgruppen Bildungstechnologien \& VR/AR, online. https:// webconf.vc.dfn.de/p75q4hy3b554?launcher=false\&fcsContent=true\&pbMode $=$ normal.

Schwan, Stephan, und Jürgen Buder. 2006. Virtuelle Realität und E-Learning. Tübingen. https://www.e-teaching.org/materialien/literatur/schwan-buder-2005.

Sherman, William R., und Alan B. Craig. 2003. Understanding Virtual Reality. Interface, Application, and Design. San Francisco, CA: Elsevier. https://doi. org/10.1016/B978-1-55860-353-0.50019-7.

Shin, Donghee. 2018. «Empathy and Embodied Experience in Virtual Environment: To What Extent Can Virtual Reality Stimulate Empathy and Embodied Experience?» Computers in Human Behavior 78 (Januar): 64-73. https://doi. org/10.1016/j.chb.2017.09.012.

Slater, Mel. 2009. «Place Illusion and Plausibility Can Lead to Realistic Behaviour in Immersive Virtual Environments». Philosophical Transactions of the Royal Society B: Biological Sciences 364 (1535): 3549-57. https://doi.org/10.1098/ rstb.2009.0138. 
Slater, Mel, Angus Antley, Adam Davison, David Swapp, Christoph Guger, Chris Barker, Nancy Pistrang, und Maria V. Sanchez-Vives. 2006. «A Virtual Reprise of the Stanley Milgram Obedience Experiments». Herausgegeben von Aldo Rustichini. PLoS ONE 1 (1): 1-10. https://doi.org/10.1371/journal.pone.0000039.

Slater, Mel, Aitor Rovira, Richard Southern, David Swapp, Jian J. Zhang, Claire Campbell, und Mark Levine. 2013. «Bystander Responses to a Violent Incident in an Immersive Virtual Environment». Herausgegeben von Frank Krueger. PLoS ONE 8 (1): e52766. https://doi.org/10.1371/journal.pone.0052766.

Slater, Mel, und Maria V. Sanchez-Vives. 2016. «Enhancing Our Lives with Immersive Virtual Reality». Frontiers in Robotics and AI 3 (Dezember). https://doi. org/10.3389/frobt.2016.00074.

Slater, Mel, Bernhard Spanlang, und David Corominas. 2010. "Simulating Virtual Environments within Virtual Environments as the Basis for a Psychophysics of Presence». ACM Transactions on Graphics 29 (4): 1. https://doi. org/10.1145/1778765.1778829.

Slater, Mel, und Sylvia Wilbur. 1997. «A Framework for Immersive Virtual Environments (FIVE): Speculations on the Role of Presence in Virtual Environments». Presence: Teleoperators and Virtual Environments 6 (6): 603-16. https:// doi.org/10.1162/pres.1997.6.6.603.

Spanlang, Bernhard, Torsten Fröhlich, Vanessa F Descalzo, Angus Antley, und Mel Slater. 2007. «The Making of a Presence Experiment: Responses to Virtual Fire». Presence: Teleoperators and Virtual Environments, 303-7.

Sutherland, Ivan E. 1968. «A Head-Mounted Three Dimensional Display». In Proceedings of the December 9-11, 1968, Fall Joint Computer Conference, Part I on - AFIPS '68 (Fall, Part I), 757. San Francisco, California: ACM Press. https://doi. org/10.1145/1476589.1476686.

Tao Ni, G.S. Schmidt, O.G. Staadt, M.A. Livingston, R. Ball, und R. May. 2006. «A Survey of Large High-Resolution Display Technologies, Techniques, and Applications». In IEEE Virtual Reality Conference (VR 2006), 223-36. Alexandria, VA, USA: IEEE. https://doi.org/10.1109/VR.2006.20.

Virtual Reality Society. 2013. «VPL Research Jaron Lanier». Virtual Reality Society (blog). 8. September 2013. https://www.vrs.org.uk/virtual-reality-profiles/vplresearch.html.

Wang, Peng, Peng Wu, Jun Wang, Hung-Lin Chi, und Xiangyu Wang. 2018. «A Critical Review of the Use of Virtual Reality in Construction Engineering Education and Training». International Journal of Environmental Research and Public Health 15 (6): 1204. https://doi.org/10.3390/ijerph15061204.

Wössner, Stephanie. 2019. «Immersives Fremdsprachenlernen». Computer+Unterricht 114: 28-31.

Zender, Raphael, Pia Sander, Matthias Weise, Miriam Mulders, Ulrike Lucke, und Michael Kerres. 2019. «Action-oriented Learning in a VR Painting Simulator.» In Proceedings of the 4th International Symposium on Emerging Technologies for Education, 2019. Magdeburg. 